A digital lock-in upgrade of the motional Stark effect diagnostic on DIII-D

J. D. King, M. A. Makowski, C. T. Holcomb, S. L. Allen, R. Geer, W. H. Meyer, D. N. Hill, D. Pham, E. C. Morse

July 19, 2010

High Temperature Plasma Diagnostics Wildwood, NJ, United States May 16, 2010 through May 20, 2010 
This document was prepared as an account of work sponsored by an agency of the United States government. Neither the United States government nor Lawrence Livermore National Security, LLC, nor any of their employees makes any warranty, expressed or implied, or assumes any legal liability or responsibility for the accuracy, completeness, or usefulness of any information, apparatus, product, or process disclosed, or represents that its use would not infringe privately owned rights. Reference herein to any specific commercial product, process, or service by trade name, trademark, manufacturer, or otherwise does not necessarily constitute or imply its endorsement, recommendation, or favoring by the United States government or Lawrence Livermore National Security, LLC. The views and opinions of authors expressed herein do not necessarily state or reflect those of the United States government or Lawrence Livermore National Security, LLC, and shall not be used for advertising or product endorsement purposes. 


\title{
A digital lock-in upgrade of the motional Stark effect diagnostic on DIII-D ${ }^{\text {a) }}$
}

\author{
J.D. King,,${ }^{1,2, b, c)}$ M.A. Makowski, ${ }^{1}$ C.T. Holcomb,${ }^{1}$ S.L. Allen, ${ }^{1}$ R. Geer,${ }^{1}$ \\ W.H. Meyer, ${ }^{1}$ D.N. Hill, ${ }^{1}$ D. Pham ${ }^{3}$ and E.C. Morse ${ }^{2}$ \\ ${ }^{1}$ Lawrence Livermore National Laboratory, Livermore, California USA \\ ${ }^{2}$ University of California-Berkeley, Berkeley, California USA \\ ${ }^{3}$ General Atomics, P.O. Box 85608, San Diego, California 92186-5608 USA
}

(Presented XXXXX; received XXXXX; accepted XXXXX; published online XXXXX)

(Dates appearing here are provided by the Editorial Office)

\begin{abstract}
Use of lock-in amplifiers for phase sensitive detection (PSD) of motional Stark effect (MSE) diagnostic signals is of critical importance to real-time internal current profile measurements in tokamak plasmas. A digital lock-in (DLI) upgrade utilizing field programmable gate array (FPGA) firmware has been installed on the MSE system of the DIII-D tokamak for eventual replacement of largely obsolete analog units. While the new digital system has shown a small reduction in electronic noise over the analog, the main advantages are reduced cost, hardware simplicity, compact size and phase tracking during plasma operations. DLI recovery of MSE polarization angles was accomplished through use of reference processing to produce only PEM $2^{\text {nd }}$ harmonic frequencies and electronic signal processing to maximize the fidelity of the recovered signal. A simplified discrete analytical solution was found that accurately describes the new DLI hardware. The DLI algorithm was found to cause a prohibitively large oscillating artifact atop the demodulated signal. The artifact was caused by the accumulator interval not containing an exact integer number of photoelastic modulator (PEM) multiplier periods. Successful MSE measurements require the minimization of this oscillating artifact amplitude. The analytical solution was used to select an appropriate accumulator interval that both reduces the artifact and maintains the greatest temporal resolution possible. Sample EFIT equilibria reconstructions, and corresponding safety factor profiles showed very close agreement between the analog and digital lock-ins.
\end{abstract}

\section{INTRODUCTION}

Digital lock-in (DLI) detection ${ }^{1}$ has become a wellestablished technique for recovering AC signals many times smaller than noise. Specifically, DLI detection employing field programmable gate arrays (FPGA) has enabled significant versatility in multi-channel systems at extremely low cost. ${ }^{2}$ While the concept of the DLI is far from new, ${ }^{3}$ implementation gives rise to application-specific subtleties. This work describes the unique DLI requirements of the motional Stark effect (MSE) diagnostic for internal magnetic field measurements of a tokamak plasma.

A new data acquisition system, installed on five operating MSE polarimeters of the DIII-D tokamak, is composed of four D-tAcq multi-channel DLI amplifiers utilizing FPGA firmware, as well as four 32-channel $500 \mathrm{kHz}$ digitizers. The digitizers record the raw photomultiplier tube (PMT) signal allowing full MSE spectral analysis of magnetohydrodynamic fluctuations ${ }^{4}$ and will not be the focus of this work. The DLI serves an identical function as previous analog lock-ins, ${ }^{2}$ recovering in real time the amplitudes of the PEM $2^{\text {nd }}$ harmonics and thus the linear polarization angle. The MSE polarimetry system and DIII-D viewing angle geometries are explained in Refs. 5-8.

\section{MSE DIGITAL LOCK-IN}

Typical MSE measurements of the polarization angle $\gamma$ utilize single phase analog lock-in amplifiers to recover the $\sin (2 \gamma)$ and $\cos (2 \gamma)$ polarimetry amplitudes from a modulated signal detected by a PMT. Demodulation relies on a phase sensitive detector (PSD) to multiply the modulated signal by a reference signal. ${ }^{9}$ This results in signals $A_{s 1, s 2} A_{r 1, r 2}\left[1-\cos ^{2}\left(2 \pi f_{1,2} t\right)\right]$, where $A_{s 1, s 2}, A_{r 1, r 2}$, and $f_{1,2}$ are the signal amplitudes, reference amplitudes, and frequencies for the MSE PEM 2nd harmonics at 40 and $46 \mathrm{kHz}$, respectively. The DC term, proportional only to $A_{s 1, s 2}$ and $A_{r 1, r 2}$, is recovered by filtering the PSD signal through a series of active low pass electronic filters. Identically, this multiplication and filtering can be done discretely using a digital system. ${ }^{1,10}$

The D-tAcq hardware serves the mixing and filtering function of the DLI. Prior to demodulation the signals and references are electronically processed. A schematic of the entire DLI hardware implementation is shown in Fig. 1.

The references originate at the PEM controllers and are sent via fiber optic transmitters from the DIII-D experimental hall to the MSE detection room. The result is a square wave reference of arbitrary duty cycle and DC offset. In order to demodulate only the desired $2^{\text {nd }}$ harmonics all other harmonics of the reference square wave must be removed. This was done by phase locking the reference to an output sinusoid of a Hewlett Packard (HP) 3314A function generator. This produced a nearly perfect sinusoid reference at the exact $f_{1}$ and $f_{2}$ frequencies. The amplitude and phase of these processed references were then adjusted to peak the DLI output. In particular, the phase differences between

${ }^{a}$ Contributed paper published as part of the Proceedings of the 18th Topical Conference on High-Temperature Plasma Diagnostics, Wildwood, New Jersey, May, 2010.

${ }^{b)}$ Electronic mail: kingjd@fusion.gat.com

${ }^{c}$ Current residence General Atomics, San Diego, California. 


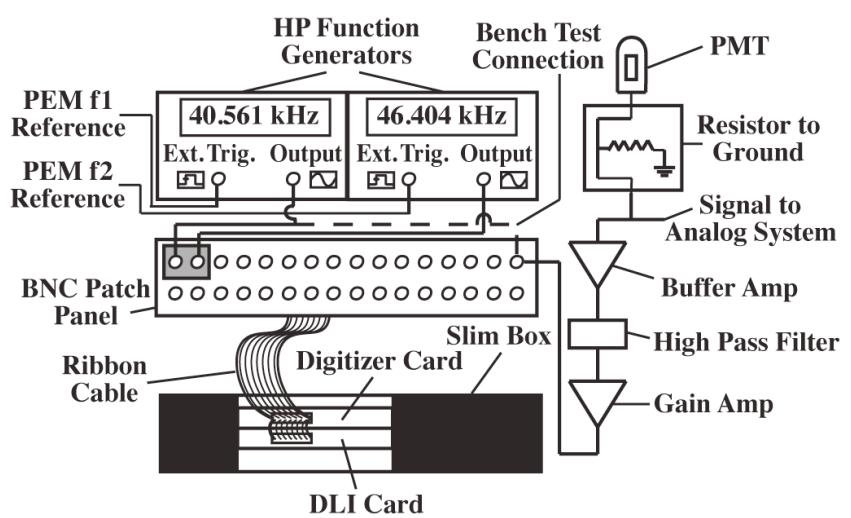

FIG. 1. Schematic of new MSE data acquisition system showing: signal processing hardware consisting of a buffer amplifier, passive high pass filter, and gain amplifier; two reference processing Hewlett Packard 3314A function generators; and the D-tAcq hardware consisting of a BNC patch panel, twisted pair ribbon cable connections to the digitizer and DLI cards, and a slim box housing. A dashed line shows the DLI bench testing connection.

the references and signals were adjusted to $0^{\circ}$ using the HP $3314 \mathrm{~A}$ phase shifter. This is possible because the raw signals and references are separately digitized and their phases can be directly recovered through Fourier analysis. Because this system permits exact phase matching and easy monitoring of the phase evolution during plasma operations, it presents a clear advantage to the single phase analog setup, which requires time consuming invessel calibrations to re-peak signals.

The electronic processing of the signal begins with the modulated MSE PMT current being transformed into a voltage drop across a $20 \mathrm{k} \Omega$ resistor connected to ground. This modulated voltage signal then passes to both the signal processing hardware of the new data acquisition system and the analog lock-in amplifiers.

Tandem operation of the new hardware and analog systems without electrical interference was needed to verify successful DLI operation. This electrical isolation was achieved by installing a very low noise unity gain Burr Brown (BB) OPA627 buffer amplifier as the first element in a signal processing chain.

The fidelity of the digitized signal is improved by utilizing the full $\pm 10 \mathrm{~V}$ dynamic range of the 16-bit digitizers. Typical MSE signals detected by the PMT contain a large DC component resulting from unpolarized background light. This DC component contains no information about the polarization and is therefore removed through a passive high pass electronic filter. This AC coupled signal allows the gain stage amplifier (also, BB OPA627) to amplify only the modulated portion of the signal to the full dynamic range of the digitizer.

The processed signals and references are connected to a BNC patch panel and transmitted to the DLI and digitizer computer cards via a twisted pair ribbon cable. Both the DLI and digitizer cards are housed in a slim box chassis with a back panel Ethernet network connection that transmits the digitized data to be stored in memory.

Once the processed signals and references arrive at the D-tAcq cards they are first digitized at $500 \mathrm{kHz}$. Then the firmware algorithm multiplies the digitally sampled signal $S(n, m)$ with the sampled sinusoidal references $R_{1,2}(n, m)$ and then sums these values over a predefined accumulator interval $L$ to filter out carrier frequencies, such that the lock-in outputs $X_{1,2}(n)$ become,

$$
X_{1,2}(n)=\sum_{m=1}^{L} S(n, m) \cdot R_{1,2}(n, m) \text {. }
$$

The subscript $n$ indexes the values of the lock-in outputs in time, and the subscript $m$ denotes the local accumulation operation. Both $n$ and $m$ are positive integers.

For the MSE DLI we have chosen to digitize the PMT signal once and demodulate the $f_{1}$ and $f_{2}$ components using separately digitized references. While the full spectrum of the MSE contains many PEM harmonics as well as beat frequencies, the following analysis was simplified by only considering the $2^{\text {nd }}$ harmonics. This means the digitized signal takes the form

$$
\begin{aligned}
S(n, m) & =A_{s 1} \cos \left(2 \pi f_{1} d t[(n-1) L+m]\right) \\
& +A_{s 2} \cos \left(2 \pi f_{2} d t[(n-1) L+m]+\phi\right),
\end{aligned}
$$

where $d t$ is the time between samples and $\phi$ is an arbitrary phase difference between the $f_{1}$ and $f_{2}$ components. The sampled references of the $f_{1}$ and $f_{2} 2^{\text {nd }}$ harmonics are,

$$
R_{1,2}(n, m)=A_{r 1, r 2} \cos \left(2 \pi f_{1,2} d t[(n-1) L+m]\right)
$$

By inserting Eq. (2) and Eq. (3) into Eq. (1) a unique analytical solution was found. For the $f_{1}$ reference this solution takes the form

$$
X_{1}(n)=\frac{A_{r 1}}{4}\left(\begin{array}{l}
A_{s 1} \csc \left(2 \pi f_{1} d t\right)\left[2 L \sin \left(2 \pi f_{1} d t\right)+\sin \left(2 \pi f_{1} d t C_{1}\right)-\sin \left(2 \pi f_{1} d t C_{2}\right)\right]+ \\
A_{s 2} \csc \left(\pi\left(f_{1}+f_{2}\right) d t\right)\left(\sin \left(\pi\left(f_{1}+f_{2}\right) d t C_{1}+\phi\right)-\sin \left(\pi\left(f_{1}+f_{2}\right) d t C_{2}+\phi\right)\right)+ \\
A_{s 2} \csc \left(\pi\left(f_{1}-f_{2}\right) d t\right)\left(\sin \left(\pi\left(f_{1}-f_{2}\right) d t C_{1}-\phi\right)-\sin \left(\pi\left(f_{1}-f_{2}\right) d t C_{2}-\phi\right)\right)
\end{array}\right),
$$

where $C_{1}=1+2 n L, C_{2}=1-2 L+2 n L$. From this expression one can readily change parameters to those of the MSE experiment, enabling quick analysis of various attributes of the algorithm.

A number of bench tests were carried out, which insured the analytical solution of Eq. (4) correctly represents the new hardware. The first bench test used the setup seen in Fig. 1, where the $f_{1}$ reference was also connected to the signal input of the patch panel instead of the modulated signal. The accumulator $L$ was arbitrarily set to 125 data points per lock-in output value, which for the $500 \mathrm{kHz}$ sample rate of the digitizer corresponds to a $4 \mathrm{kHz}$ data throughput rate for the DLI. These DLI parameters, along with the signal frequency of $40.561 \mathrm{kHz}$ and amplitudes
$A_{s 1} \approx A_{r 1} \approx 4 V$ and $A_{s 2} \approx A_{r 2} \approx 0 V$ where entered into Eq. (4). In Fig. 2 it can be seen that the analytical solution agrees with the DLI data both for the overall DC recovered amplitude, and the fine oscillating structure after an arbitrary phase shift is applied to the result.

\section{OSCILLATING ARTIFACT}

The oscillating artifact seen in Fig. 2 is not acceptable for real-time MSE polarization angle $\gamma$ measurements. In an initial plasma data comparison with the analog lock-ins the DLI, with accumulator 125 , showed a factor of 5 greater variation in the recovered $\gamma$, due to this oscillation. 


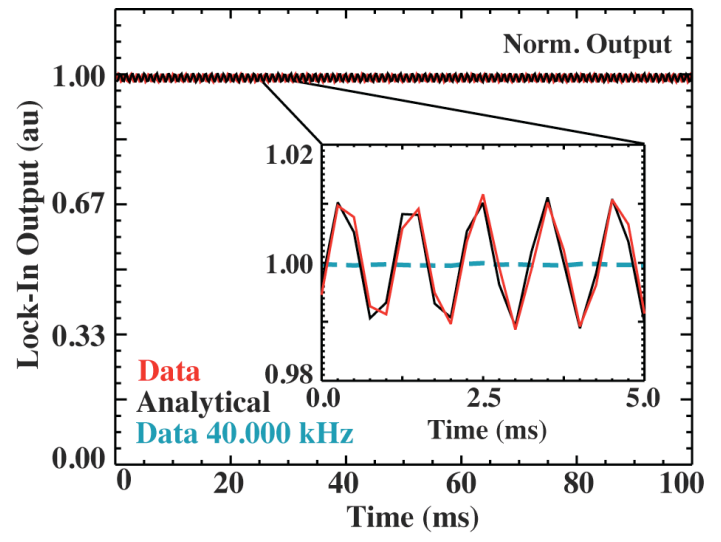

FIG. 2. Red represents the DLI output for a synthetic function generator signal input at the PEM frequency. Black represents the analytical solution computed for the same input parameters as the red synthetic signal. Blue represents the normalized DLI output for a $40.000 \mathrm{kHz}$ synthetic input.

This artifact is caused when the accumulator interval does not contain an exact integer number of multiplier output periods. For the example shown in Fig. 2 the dimensionless expression $f_{l} d t L$ must be an integer to avoid the oscillating artifact. Note, when $f_{l} d t L$ and $f_{2} d t L$ are integer values, Eq. (4) simplifies to an expression containing only the desired DC value proportional to the signal amplitude and accumulator interval,

$$
X_{1}=\frac{A_{s 1} A_{r 1} L}{2} .
$$

Further confirmation of this non-integer multiple effect was found by adjusting the function generator frequency of the DLI input signal and reference to $f=40.000 \mathrm{kHz}$. The accumulator parameter $L$ was again set to 125 points, and $d t=0.002 \mathrm{~ms}$. These DLI parameters give an integer value for $f_{1} d t L$ of 5 , and result in the complete elimination of the oscillating artifact seen in the normalized output of Fig. 2. Note that this test only considers a single signal frequency. Including an additional signal at $f_{2}$ with a non-integer value for $f_{2} d t L$ would cause another artifact to appear on the $f_{1}$ DLI output.

Tuning the PEM frequencies such that $f_{1} d t L$ and $f_{2} d t L$ are integer values is not possible. The frequencies of the PEMs

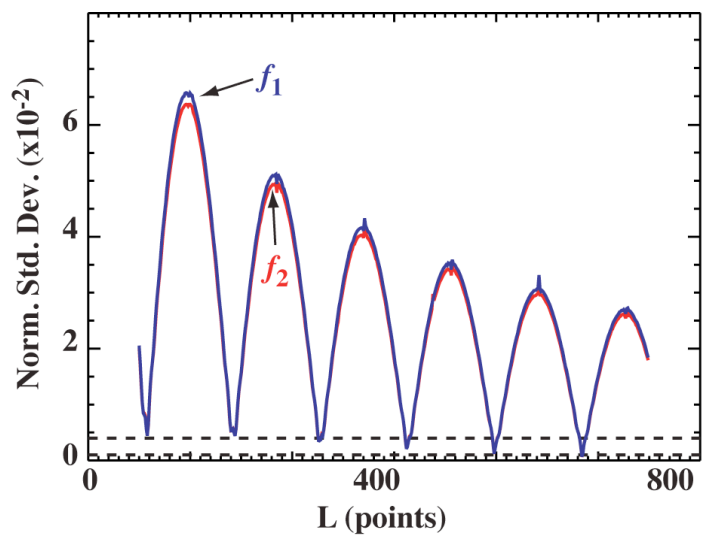

FIG. 3. Blue line represents analytically calculated amplitude of the oscillating artifact for $f_{1}$, and red line represents the same result for $f_{2}$. Dotted lines show the region of analog lock-in electronic noise. are inherent to the crystal dimensions and, to a lesser degree, environmental conditions. For example, the tangential MSE PEMs have $2^{\text {nd }}$ harmonic frequencies of 40.561 and $46.404 \mathrm{kHz}$, and these frequencies slowly change by 7 to $10 \mathrm{~Hz}$ over a given run day as temperatures in the DIII-D experimental hall vary. Therefore, the only adjustable parameter of the $f_{1} d t L$ and $f_{2} d t L$ expressions is the accumulator interval $L$.

The firmware of the DLI allows the accumulator $L$ to be set to any integer value between 2 and 5000 . By varying $L$ between 250 and 750 in Eq. (4), and setting $A_{s 1}, A_{s 2}, A_{r 1}$, and $A_{r 2}$ to about $4 \mathrm{~V}$ an identical trend in the standard deviation of the artifact was found for both the $f_{1}$ and $f_{2}$ DLI outputs. This trend is shown in Fig. 3. In addition to the general trend of decreasing artifact amplitude with increasing $L$, the standard deviation of the artifact approaches zero at six nodes (e.g. $L \sim 431$ ). Each of these nodes correspond to $L$ values that allow both of the $f_{1} d t L$ and $f_{2} d t L$ expressions to approach integer values. For example, at the $L=431$ node the $f_{1} d t L$ and $f_{2} d t L$ expressions approach integers 35 and 40, respectively. Also, the nodes $L$ values were found to be invariant with respect to $\phi, A_{s 1, s 2}$ and $A_{r 1, r 2}$. However, the overall standard deviation of the artifact does vary when the ratio of the signal amplitudes is changed.

The $L=431$ node was found to be the smallest accumulator interval that reduces the DLI oscillating artifact beneath the electronic noise $\left(1 \sim 4 \times 10^{-3}\right.$ in Fig. 3) of the analog system for both the $f_{1}$ and $f_{2}$ signals. At a temporal resolution of $\sim 0.9 \mathrm{~ms}$, this accumulator is comparable to the $2 \mathrm{kHz}$ rate at which the analog units are sampled.

Recall, the simplifying assumption that the MSE spectrum only contains $2^{\text {nd }}$ harmonic components. For our setup the $2^{\text {nd }}$ harmonic amplitudes are about an order of magnitude greater than the odd harmonics and beat frequency amplitudes. It is likely that this $2^{\text {nd }}$ harmonic dominated spectrum allows this simplified analysis to successfully characterize the artifact reduction. However, Eq. (4) may no longer be representative of the DLI output if other harmonics and beats have a greater proportion in the spectrum.

\section{SAMPLE MEASUREMENT}

By setting $L$ to 431 for the tangential MSE array, the DLI recovered $\gamma$ now matches that of the analog system for all channels. A few examples of this agreement are shown in Fig. 4. The channel 7 standard deviations from $\sim 3800$ to $4000 \mathrm{~ms}$ were $0.12^{\circ}$

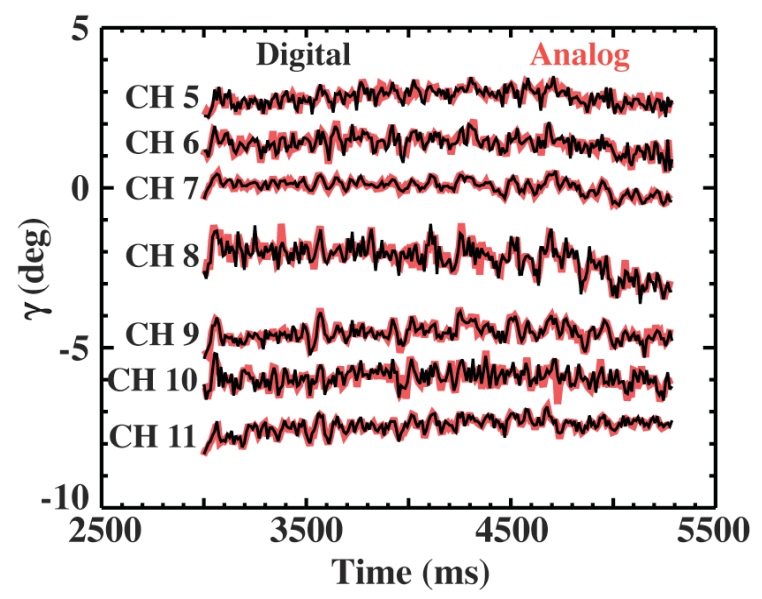

FIG. 4. Black represents DLI-recovered $\gamma$. Red/gray represents analog lock-in recovered $\gamma$ (shot 142349). 
and $0.10^{\circ}$ for the analog and DLI, respectively. The difference in averaged $\gamma$ values for both systems across all channels was less than the $0.1^{\circ}-0.2^{\circ}$ uncertainty of our measurement. $^{6}$

Using DLI-recovered $\gamma \mathrm{s}$, EFIT ${ }^{11}$ equilibria were computed and showed very good overall agreement with equilibria computed using analog data. Figure 5 compares the equilibria of the analog and DLI for shot 142349 at $4000 \mathrm{~ms}$. The magnetic axis of both the analog and DLI were computed to be $3.6 \mathrm{~cm}$ below the machine midplane. The major radius of the magnetic axis showed only a $3 \mathrm{~mm}$ difference between the two cases.

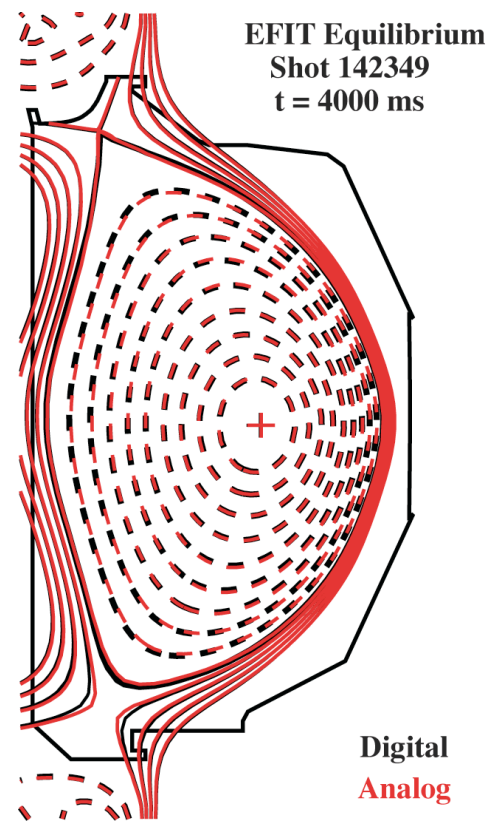

FIG. 5. Black represents the EFIT equilibrium using DLI data. Red/gray represents an EFIT equilibrium from analog lock-in data (shot 142349).
Also, significant agreement of the DLI- and analogcomputed safety factor profile was observed. Both systems show $q$-min $\sim 1.1$, with only $5 \%$ difference between them. The safety factor at normalized $\rho$ of 0.95 shows only a $3 \%$ difference. The internal inductance measured by both systems was $\sim 0.7$.

These observations demonstrate, with careful consideration of the accumulator interval, this compact, inexpensive, and simple multi-channel DLI can provide an identical real-time MSE measurement to its analog counterpart.

\section{ACKNOWLEDGMENT}

This work was performed under the auspices of the US DOE by Lawrence Livermore National Laboratory under DE-AC5207NA27344, and US DOE DE-FC02-04ER54698 and DE-FG0389ER51116.

${ }^{1}$ E. D. Morris, Jr. and H. S. Johnston, Rev. Sci. Instrum. 39, 620 (1968).

${ }^{2}$ A. Restelli, R. Abbiati, and A. Geraci, Rev. Sci. Instrum. 76, 093112 (2005).

${ }^{3}$ L. G. Rubin, Rev. Sci. Instrum. 59, 514 (1988).

${ }^{4}$ R. J. Jayakumar, M. A. Makowski, S. L. Allen, M. E. Austin, A. M. Garofalo, R. J. La Haye, H. Reimerdes, T. L. Rhodes, Rev. Sci. Instrum. 75, 9 (2004).

${ }^{5}$ F. M. Levinton, R. J. Fonck, G. M. Gammel, R. Kaita, H. W. Kugel, E. T. Powel, and D. W. Roberts, Phys. Rev. Lett. 63, 19 (1989).

${ }^{6}$ D. Wroblewski, K. H. Burrell, L. L. Loa, P. Politzer, and W. P. West, Rev. Sci. Instrum. 61, 3552 (1990).

${ }^{7}$ B. W. Rice, D. G. Nilson, and D. Wroblewski, Rev. Sci. Instrum. 66, 373 (1994).

${ }^{8}$ C. T. Holcomb, M. A. Makowski, R. J. Jayakumar, S. L. Allen, R. M. Ellis, R. Geer, D. Behne, K. L. Morris, L. G. Seppala, and J. M. Moller, Rev. Sci. Instrum. 77, 10E506 (2006).

${ }^{9}$ Stanford Research Systems, About Lock-In Amplifiers, Application Note 3, http://www.thinksrs.com/downloads/PDFs/ApplicationNotes/ AboutLIAs.pdf

${ }^{10}$ S. J. Lascos and D. T. Cassidy, Rev. Sci. Instrum. 79, 074702 (2008).

${ }^{11}$ L. L. Lao, J. R. Ferron, R. J. Groebner, W. Howl, H. St. John, E. J. Strait, T. S. Taylor, Nucl. Fusion 30, 6 (1990). 